\title{
Sagesse et résistance dans les littératures francophones, dir. M. QUAGHEBEUR avec la collaboration de N. LECLERCQ
}

\section{Elena Fermi}

\section{(2) OpenEdition}

\section{Journals}

\section{Édition électronique}

URL : https://journals.openedition.org/studifrancesi/23269

DOI : $10.4000 /$ studifrancesi.23269

ISSN : 2421-5856

\section{Éditeur}

Rosenberg \& Sellier

\section{Édition imprimée}

Date de publication : 1 avril 2020

Pagination : 220-222

ISSN : 0039-2944

\section{Référence électronique}

Elena Fermi, « Sagesse et résistance dans les littératures francophones, dir. M. QUAGHEBEUR avec la collaboration de N. LeCLerca », Studi Francesi [En ligne], 190 (LXIV | I) | 2020, mis en ligne le 01 mai 2020, consulté le 02 août 2021. URL : http://journals.openedition.org/studifrancesi/23269; DOI : https://doi.org/10.4000/studifrancesi.23269

Ce document a été généré automatiquement le 2 août 2021.

\section{(i) $९$

Studi Francesi è distribuita con Licenza Creative Commons Attribuzione - Non commerciale - Non opere derivate 4.0 Internazionale. 


\title{
Sagesse et résistance dans les littératures francophones, dir. M. QUAGHEBEUR avec la collaboration de N. LECLERCQ
}

\author{
Elena Fermi
}

\section{RÉFÉRENCE}

Sagesse et résistance dans les littératures francophones, dir. M. QUAGHEBEUR avec la collaboration de N. LECLERCQ, Bruxelles, Peter Lang, 2018, 636 pp.

1 Ce volume fait partie de la collection «Documents pour l'Histoire des Francophonies» qui, publiée avec l'aide des Archives et Musée de la Littérature et soutenue par la Fédération Wallonie-Bruxelles, entend mettre à la disposition des lecteurs des études critiques touchant à la complexité comme aux enracinements historiques des Francophonies. Son objectif principal est celui d'ouvrir le devenir du français à la pluralité des littératures francophones, qui ont bénéficié, à partir des dernières décennies $d u x^{e}$ siècle, d'une reconnaissance en tant que telles. Le sujet qui va être traité de manière transversale par les différentes contributions faisant partie de cet ouvrage est celui de la résistance «littéraire» à l'oppression physique ou intellectuelle. Un volume de 2013 s'était déjà posé la question et l'avait abordée du point de vue de la réponse violente donnée par les intellectuels à cette problématique. Ce recueil complète l'enquête, en abordant le sujet à partir d'un autre versant, celui «des sagesses qui découlent de formes de révolte et de résistance parfois tout aussi obstinées» (p. 13). Les champs des Francophonies explorés par les différents contributeurs sont nombreux et vont de l'Europe aux Amériques, de l'Afrique à l'Asie. Le présent compte-rendu ne va prendre en compte que les études concernant les expressions littéraires non 
européennes, en laissant de côté celles qui ont été produites en France, en Suisse ou bien en Belgique.

Bernadette DESORBAY ouvre les danses avec son essai intitulé Opacités francophones sagesse post-senghorienne. Résistance à la France/Métropole et aux monopoles de la conscience. Elle y aborde la problématique de la résistance par le biais d'un discours qui prend en compte la psychanalyse. À travers un corpus d'œuvres qui représentent la complexité et la variété de l'univers francophone, ainsi que l'évolution de l'idée de Francophonie au cours des décennies, elle met l'accent sur la perte d'influence de la France/ Métropole et de la Belgique par rapport aux anciennes colonies qui ont développé leur propre discours de résistance en allant au-delà de la domination qui passait par l'imposition du français comme langue d'expression privilégiée. À partir du discours des fondateurs de l'anticolonialisme et du mouvement de la Négritude - Senghor et Césaire en particulier - pour arriver à Glissant et Chamoiseau mais aussi à des auteurs tels qui Biyaoula et Ngangan, l'auteure met l'accent sur la nécessité qu'a la littérature de créer son propre langage, un langage qui tienne compte de l'opacité qui pourrait le caractériser et qui en fasse ressortir les mots de l'inconscient.

La première section du volume, intitulée «Quand la résistance à l'Histoire devient sagesse», présente un article de Brigitte DoDu consacré à Des Marrons et des Batoutos. Le peuple inverse d'Édouard Glissant. Elle y aborde la pensée glissantienne concernant le Tout-Monde, à travers un corpus qui convoque La Cohée du Lamentin, cinquième de ses Poétiques et Sartorius: le roman des Batoutos, où il imagine la conjonction des Hommes et d'un «peuple inventé», les Batoutos, confronté au choix entre mondialité et mondialisation. L'étude de l'évolution de la pensée glissantienne et la mise en relief de ses constantes stylistiques et narratives mènent l'auteure à formuler l'hypothèse d'une gémellité entre l'intellectuel martiniquais et Saint-John Perse. Elle aborde par ailleurs l'œuvre de Glissant par le biais de l'utopie humaniste dont elle se fait porteuse. Kathleen GysSELS consacre sa contribution à "Resouvenir» et résilience: l'écriture réversible d'André Schwarz-Bart. En analysant un corpus d'œuvres de cet écrivain et de sa femme Simone, elle met l'accent sur le double devoir de mémoire de l'oppression subie par les Juifs et par les Noirs que très peu de fictions caribéennes ont eu le courage d'affronter. Les Schwarz-Bart, qui participent de ces deux destins, montrent dans leurs œuvres «la hantise d'Auschwitz et la «réversibilité» entre le destin juif et le destin noir dans l'Univers des Plantations» (p. 141). «Leur travail sur la mémoire et l'écriture mémorielle - dit Gyssels - tisse un lien insécable entre cycle antillais et cycle ashkénaze» (p. 141), en faisant dialoguer deux peuples et deux Histoires. Quand La Nuit sauvage de Mohamed Dib fait l'objet de l'étude de Fritz Peter KIRSCH intitulée À la recherche d'une sagesse face à l'horreur. Le chercheur considère ce recueil de nouvelles publié en 1995 - dans lequel l'empreinte de la violence se manifeste avec une insistance croissante - comme une plaque tournante dans la production de l'auteur. Sa mise en scène de la violence par les mots montre - selon Kirsch - l'angoisse de l'écrivain qui s'interroge sur la portée de l'art confronté aux réalités les plus sombres de l'Histoire et qui, loin de donner des réponses, soulève des questions et formule des doutes, tout en restant convaincu de la responsabilité morale de l'écrivain. Chloé MoNEY concentre son attention sur l'œuvre de Kateb Yacine, «sage ennemi» de la France qui opprima par la torture la révolte algérienne. Pour ce faire, elle établit un dialogue entre l'écriture du poète et les faits historiques. Si les pires atrocités auxquelles la France se livra pendant la guerre d'Algérie l'obligent encore aujourd'hui à se confronter à une mémoire 
gênante, des poèmes tels que Le Cadavre encerclé contribuent à une prise de conscience des contradictions entre une histoire interne de combat contre l'oppression et la mise en œuvre de cette même oppression dans les territoires colonisés. Money évoque aussi le poème Dans la gueule du loup de 1987, dans lequel l'écrivain réagit à l'oppression des manifestations algériennes qui eurent lieu le 17 octobre 1961 en France. Elle élargit ainsi son discours au langage adopté par le poète qui, en réduisant les mots à l'état de signes vidés de sens, entend montrer la fêlure à l'origine de la trahison de la langue. Kateb Yacine, passionné par l'histoire française, tenterait avec l'écriture de s'élever au rang de redresseur des torts commis par l'ancienne métropole envers son pays, en lui rappelant constamment qu'elle a derrière elle la Commune et la Révolution. Ouatann, de l'écrivaine tunisienne Azza Filali, fait l'objet de l'intervention d'Yves CHEMLA. Dans ce roman, publié en 2012, plusieurs personnages, couvrant le spectre social tunisien, se rencontrent dans une maison dressée face à la mer, près de Bizerte. L'histoire s'articule sur plusieurs ruptures et sur un désir d'utopie progressivement délivré, ainsi que sur la question de la mémoire à laquelle les personnages donnent des réponses différentes. Selon Chemla, le lieu aurait dans ce roman - qui fait à peine percevoir les événements de la révolution tunisienne de 2010-2011 - la double fonction mémorielle et de dépassement de la mémoire. Concilie BigiRIMANA traite de la mythification de l'Histoire à travers Les Années avalanche de Juvénal Ngorwanubusa, dont elle esquisse une analyse basée sur une approche sociocritique. L'étude entend montrer que la structure sémantique et syntaxique de ce texte - sorte de réécriture de l'Histoire nationale du Burundi - se révèlent à travers l'opposition ethnique et idéologique du récit. La forme de résistance mise en œuvre par l'auteur correspond ici à une mise en scène de la stupide concurrence entre les deux ethnies principales du pays, dont il tourne en dérision les configurations absurdes tout en en glorifiant les valeurs positives.

4 La deuxième section du volume, «Une poét(h)ique de la résistance», présente un article de Fady FADEL et Cynthia EID consacré à la révolution du Cèdre au Liban. Les deux contributeurs esquissent d'abord un panorama historique des principaux événements ayant eu lieu dans le pays après les accords de Taëf qui mirent fin à la guerre civile qui avait ravagé le pays entre 1975 et 1989, en le soumettant de facto à la tutelle syrienne. Un mouvement d'opposition et de résistance à cette situation ne tarda pas à se former et amena, le 14 février 2005, à l'assassinat de Rafic Hariri, ancien président du Conseil des Ministres désormais considéré un dangereux opposant au statu quo. C'est à partir de cette date clé que les libanais organisèrent leur résistance pacifique aux ingérences de tous bords, venant des pays du Proche Orient mais aussi de la communauté internationale. La révolution du Cèdre, le 14 mars 2005, amena plus d'un million de manifestants à se rassembler pour revendiquer l'indépendance du pays. La résistance libanaise est ensuite présentée à travers ses traces littéraires; les auteurs prennent en considération La guerre m'a surprise à Beyrouth de Carmen Boustani, L'armoire des ombres de Hyam Yared et Kamal Jann de Dominique Eddé, afin de montrer comment cette résistance a été mise en scène par les écrivains qui ont osé affronter, avec leurs mots, les maux de la société, résister à l'absurde et briser les tabous. Le Congo est mis à l'honneur dans l'article de Jean-Claude KANGOMBA, Écriture et pensée unique durant les années Mobutu. Le cas de "Misère au point". L'auteur se propose d'y présenter quelques figures de la résistance à la dictature de Mobutu, l'une des plus spectaculaires - et des plus mortifères - $d u$ continent africain. Ses figures sortent toutes du milieu universitaire, auquel le dictateur s'attaque à partir de 1969, ce qui entraîne le départ en un exil souvent forcé de ces intellectuels qui souhaitent garder leur liberté. Les 
exemples littéraires qui illustrent cette situation ne manquent pas: Mudimbe la détaille dans son essai autobiographique Le corps glorieux des mots et des êtres, Pius Ngandu dans La Délivrance d'Illunga. Kangomba met au premier plan de sa contribution son propre roman de 1987 Misère au point, dans lequel il s'interroge - à partir de faits réels - sur la parole et le droit à la parole, ainsi que sur la question du discours et de son usage dans l'espace public. C'est donc d'un engagement des intellectuels et d'une résistance qui passe par la prise de parole que le contributeur parle dans son article. La littérature engagée est également à l'honneur dans l'article de Margarita ALFARO consacré à la littérature sahraouie en langue française. L'auteure se concentre en particulier sur $L a$ Plume prisonnière de Na'Ana Labbat El-Rachid, un recueil de 19 poèmes, d'abord écrits en arabe classique, puis traduits en français par la poétesse elle-même. Ces poèmes, écrits en situation d'exil, expriment, selon Alfaro, «l'angoisse existentielle de l'auteure et servent de moteur à une nouvelle dimension poétique pour la tradition hassanophone exprimée en arabe, et qui connaît sa diffusion en langue française» (p. 324). El-Rachid représente elle aussi un exemple de sagesse et de résistance à travers les mots qui exaltent son peuple et le poussent à parvenir au meilleur de son destin personnel. C'est encore de poésie que traite Cristina ROBALO-CORDEIRO, de poésie arabe contemporaine d'expression française. La question que l'auteure se pose ici est celle de la relation entre poésie et religion, notamment du point de vue du langage utilisé dans ces deux domaines. À partir de l'axiome que poésie et religion partagent le même langage, l'auteure affronte la problématique de la liberté d'expression, qui peut être atteinte par le contrôle que l'autorité exerce sur la langue. Si l'arabe classique - celui du Coran - est pénétré de religion et est donc sujet à un examen constant de la part de l'autorité, ce qui peut donc entraver les poètes, la laïcité du français permet aux poètes de trouver une terre d'asile favorisant la libre expression de la pensée. Léon-François HOFFMANN s'attache à la littérature haïtienne et se propose d'examiner la tension entre résistance et prudence qui se manifeste depuis les origines chez les hommes de lettre haïtiens. En relatant l'histoire de l'île depuis son indépendance, l'auteur remarque que les menaces aux intellectuels ne sont jamais venues de l'autorité religieuse, ni de l'autorité judiciaire - ce qui caractérise d'autres littératures de langue française - mais plutôt des dictatures qui se sont succédé. La résistance des intellectuels haïtiens s'est faite surtout de l'exil auquel beaucoup d'entre eux ont été forcés, même si les dictatures n'ont jamais vraiment mis en place une censure des écrits ni une persécution des intellectuels à cause de leurs paroles. Une forme de sagesse leur a conseillé de s'opposer au régime qui opprimait leur pays depuis l'exil étranger. Leur dénonciation des violences de la dictature est cependant très forte, prend une tonalité très personnelle et reflète le désespoir de ses victimes.

5 La troisième section du volume s'intitule «Quand l'altérité permet l'hybridation identitaire». Émilienne AKONGA s'attache au récit Sans rancune de l'écrivain congolais Thomas Kanza. À partir de ce titre qu'à la suite de Genette l'auteure définit de thématique et qui est, de fait, programmatique, le récit est analysé du point de vue de la mise en scène que l'auteur y fait d'une résistance contre la violence coloniale des Belges dans le pays. En développant des sujets tels que la fascination du voyage et de la formation en Europe, l'avenir des rapports entre Blancs et Noirs, la confrontation entre culture occidentale et africaine, ce récit - qui se nourrit de l'expérience de l'auteur représente, selon Akonga, une véritable éthique de la résistance par la sagesse africaine. L'œuvre du romancier haïtien Dany Laferrière fait l'objet de la contribution de Marie JOQUEVIEL-BOURJEA qui choisit comme corpus pour son analyse Le Cri des oiseaux 
fous et L'Énigme du retour. Son but est celui de montrer que l'éthique d'une existence libre malgré les difficultés - éthique que l'écrivain a adoptée - est une forme de sagesse qui s'explicite en une forme romanesque elle aussi foncièrement libre et que la création littéraire est, chez Laferrière, une terre d'asile qui permet d'outrepasser les événements de l'Histoire. Les écrivains haïtiens et l'histoire de l'île sont mis à l'honneur aussi par Peter KLAUS qui, à partir du roman de Marie-Célie Agnant, Un alligator nommé Rosa, traite de l'héritage des dictatures des Duvalier dans la production des écrivains de la diaspora. En mettant en scène une confrontation entre la victime et son bourreau et en inversant les rôles de l'homme, la victime, et de la femme, le bourreau, la romancière haïtienne ferait faire à ses protagonistes - selon Klaus - un travail de mémoire qui d'individuelle devient collective et qui aborde la problématique de la réparation de l'injustice tout en restant juste, sujet auquel il est évidemment très difficile de donner une réponse satisfaisante. Deux intellectuels issus de l'immigration, la vietnamienne Kim Thùy et l'iranien Philippe Blasband font l'objet de la contribution de Céline MARIAGE qui aborde leur œuvre par le biais de la quête identitaire. Selon l'auteur, ses deux écrivains mettraient en scène dans leur production littéraire la libération de l'exilé à travers l'écriture. Elle traite d'abord des trois temporalités de l'exil: l'enfance dans le pays natal, l'exil dans un autre pays, le moment de l'écriture et de la réflexion dans le pays d'accueil. Grâce à une analyse et à une confrontation de l'œuvre des deux créateurs, elle relève des similitudes frappantes: une portée qui se veut, selon elle, universelle, l'idée de prendre la parole au nom de tous les exilés, un parcours de quête identitaire douloureux livré aux lecteurs sous une forme poétique et un message d'espoir transmis à tous ceux qui ont vécu ou qui vivent la même expérience.

6 La quatrième section, «Les chemins de traverse de la résistance», présente la contribution de Abderrahmane AJBOUR concernant l'œuvre de l'écrivain marocain Mohamed Khaïr-Eddine, en particulier son récit Il était une fois un vieux couple heureux. Si le chercheur considère que cet ouvrage se distingue des autres textes de l'auteur par la nature classique de sa composition, il y relève la présence constante du thème de la résistance, une résistance qui passe par la langue - celle de l'autre car c'est du français que l'on parle - mais aussi par le refus du protagoniste d'entrer dans le jeu du système et de ses vanités. Les Résistances tunisiennes font l'objet de l'article de Samia KASSABCHARFI, qui affirme que la résistance ne serait qu'un hyponyme du concept de sagesse. Elle cite en exemple trois intellectuels qui ont mis en place, dans leur œuvre, des formes différentes de résistance: polémique chez Fawzi Mellah dans Le Conclave des pleureuses (1987), combattive chez Hélé Béji, auteure de L'œil du jour (1985), plus douce et modérée chez Emna Belhaj Yahia dans Tasharej (2000) et Jeux de rubans (2011). L'analyse que la chercheuse fait de ce corpus entend faire découvrir aux lecteurs des formes différentes de réaction à l'Histoire tunisienne via la littérature.

7 La dernière section du volume, qui aborde le sujet de «L'écriture comme mode de résistance», présente trois contributions concernant toutes des écrivains francophones européens qui ne vont donc pas faire pas l'objet du présent compte-rendu. En clôture $\mathrm{du}$ volume, les notices biographiques des contributeurs permettent de repérer des informations plus détaillées sur leur rôle au sein des institutions universitaires, ainsi que sur leurs publications principales.

8 Cet ouvrage imposant, en traitant des auteurs d'origines différentes, dresse un panorama de la question annoncée dès le titre - l'écriture comme sage moyen de 
résistance - et ouvre, ce faisant, à d'ultérieurs développements. La perspective sociocritique constitue le fil rouge autour duquel se rassemblent tous les articles présentés et permet de repérer des constantes thématiques chez les auteurs venant du même milieu historique et culturel. Si la littérature contemporaine est la protagoniste absolue et l'objet de la plupart des tractations, des échappées chez des auteurs moins récents ne manquent pas. 